\title{
Severe ileus after colonoscopy in a patient on peritoneal dialysis
}

\author{
Sang Un Kim, Su Hee Kim, So Yoon Hwang, Ryang Hi Kim, Ji-Young Choi, Jang-Hee Cho, \\ Chan-Duck Kim, Yong-Lim Kim, Sun-Hee Park \\ Division of Nephrology, Department of Internal Medicine, Kyungpook National University School of Medicine, Daegu, Korea
}

Peritoneal dialysis (PD) is associated with the development of various complications, such as exit site infection or peritonitis, and rarely, intestinal obstruction in prolonged PD patients with recurrent peritonitis. However, post-colonoscopy acute intestinal obstruction has not been reported in PD patients to date. Herein, we report a case of severe ileus after a colonoscopy without previous episodes of peritonitis in a PD patient. A 51-yearold man undergoing PD for 7 years visited our emergency department due to severe abdominal pain and vomiting after colonoscopic polypectomy. A simple abdominal radiography and abdominal computed tomography showed ileus with collapsed distal ileal loop. A peritoneal dialysate study revealed no evidence of peritonitis. The patient was treated with decompression therapy, and ileus was successfully treated without complications. This case suggests that it is not only necessary to prevent peritonitis, but also important to monitor the development of ileus after colonoscopy in PD patients.

Keywords: Colonoscopy; lleus; Peritoneal dialysis

\section{INTRODUCTION}

Colonoscopy is commonly performed to diagnose and treat colorectal diseases in patients with gastrointestinal symptoms. Complications most commonly reported are perforation and hemorrhage after polypectomy [1]. Unusual but serious complications related to colonoscopy - splenic trauma, colon volvulus, small bowel perforation, and small bowel obstruction - have been reported to date [2].

Several reports have demonstrated the possibility to develop peritonitis after a colonoscopy for patients on peritoneal dialysis (PD) [3]. Since peritonitis after colonoscopy may lead to

Received: May 24, 2016, Revised: July 15, 2016

Accepted: August 9, 2016

Corresponding Author: Sun-Hee Park, Division of Nephrology, Department of Internal Medicine, Kyungpook National University School of Medicine, 130 Dongdeok-ro, Jung-gu, Daegu 41944, Korea

Tel: +82-53-420-5547, Fax: +82-53-421-5547

E-mail: sh-park@knu.ac.kr serious morbidity, the guidelines set forth by the International Society for Peritoneal Dialysis recommend antibiotic prophylaxis prior to a colonoscopy in PD patients [4]. However, to date, acute intestinal obstruction after a colonoscopy has not been reported in stable PD patients. Herein, we report a case of severe ileus without evidence of peritonitis after a colonoscopy in a patient on PD.

\section{CASE}

A 51-year-old man on automatic PD for 7 years visited our colorectal clinic with hematochezia. He has been on PD without episode of peritonitis since the initial placement of the peritoneal catheter by a surgical technique in 2008 . The initial peritoneal equilibrium test revealed that the dialysate-to-plasma creatinine ratio (D/Pcr) was 0.765 (high average), and it was maintained in the same category of membrane transport characteristics until 1 year before the onset of this event. He used Baxter HomeChoice PD Automated Cycler Machine

Copyright $($ C) 2017 Yeungnam University College of Medicine

This is an Open Access article distributed under the terms of the Creative Commons Attribution Non-Commercial License (http://creativecommons.org/licenses/by-nc/4.0/) which permits unrestricted non-commercial use, distribution, and reproduction in any medium, provided the original work is properly cited. 
(Baxter Ltd., Singapore). The cycler prescription was for 9 hours, which was composed of 4 cycles of 2.5 liters (glucosebased PD solution) of dwell volume with 2 liters of icodextrin as the last fill. Weekly Kt/V urea was 1.77 . His comorbidities were hypertension and diabetes. His medication included anti-hypertensive drugs, proton pump inhibitor, statin, antihistamine, calcium acetate, and monthly exogenous erythropoietin. The medication did not change for 7 months before this visit. Except laparoscopic PD catheter insertion, the patient had no history of intestinal surgery, hernia, constipation, or intestinal obstruction.

A colonoscopy was performed by a trained endoscopist to investigate the cause of hematochezia and also as a routine screening given his age. He had a history of hemorrhoids, which were suspected to cause hematochezia. The bowel was prepared with polyethylene glycol electrolytes solution. Intravenous midazolam was injected for sedation, and third-generation cephalosporin was administered as a prophylactic antibiotic before the colonoscopy. Colonoscopic findings included nine polyps, all of which were removed in a 1.5 -hour period without any complication. Histological findings revealed that the polyps were tubular adenomas. Internal hemorrhoids were confirmed as the cause of hematochezia.

On the day after the procedure, he suffered abdominal distension, abdominal pain, bilious vomiting, and no passage of intestinal gas. He visited a local medical center and was treated conservatively, but his symptoms persisted without improvement. Eventually, he was transferred to our emergency department 5 days after the colonoscopy. On physical examination, his abdomen was markedly distended, tympanic, and diffusely tender with decreased bowel sounds. His vital signs were stable. The laboratory analyses were as follows: white blood cell, 14,920/ $\mu \mathrm{L}$; hemoglobin, $11.7 \mathrm{~g} / \mathrm{dL}$; platelet count, 441,000/ $/ \mathrm{L}$; sodium, $130 \mathrm{meq} / \mathrm{L}$; potassium, 4.4 meq $/ \mathrm{L}$; calcium, $8.7 \mathrm{mg} / \mathrm{dL}$; phosphate, $5.1 \mathrm{mg} / \mathrm{dL}$; blood urea nitrogen, $34.1 \mathrm{mg} / \mathrm{dL}$; creatinine $4.4 \mathrm{mg} / \mathrm{dL}$; and C-reactive protein, $3.1 \mathrm{mg} / \mathrm{dL}$. PD fluid examination revealed $25 / \mu \mathrm{L}$ of white blood cells with $100 \%$ lymphocytes.

An abdominal plain film showed a typical pattern of ileus with multiple loops of dilated small bowel (Fig. 1). An abdominal computed tomography (CT) showed ileus with a fluid-filled small bowel and collapsed distal ileal loop (Fig. 2). The PD catheter tip was normally located in the pelvic cavity.

He was treated conservatively with decompression using the nasogastric tube, bowel rest, intravenous fluids, and prophylactic antibiotic for 8 days. Ileus was relieved without any complications, and a follow-up abdominal radiography showed stepwise interval resolution (Fig. 3). On the 8th day
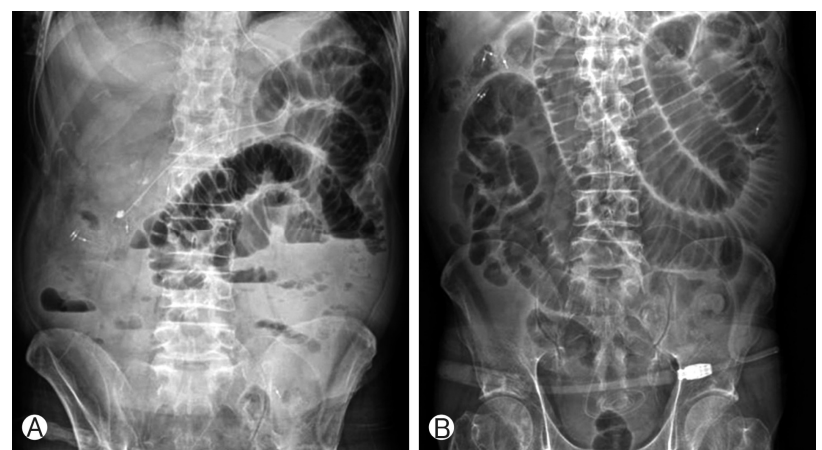

Fig. 1. Simple abdominal radiograph, supine (A) and erect (B); the abdomen shows diffuse dilated loops of gas-filled small bowel.

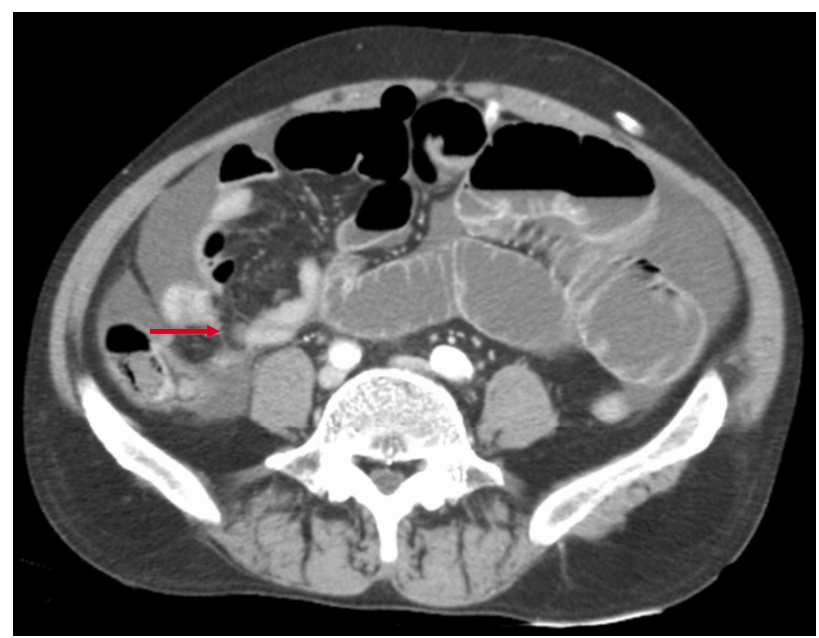

Fig. 2. Abdominal computed tomography shows ileus with fluidfilled small bowel with collapsed distal ileal loop (red arrow).

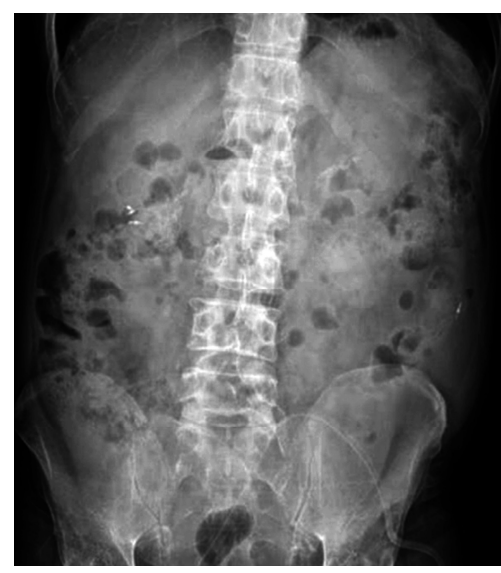

Fig. 3. Simple abdominal radiograph findings. Erect abdomen radiograph eight days after treatment (on the day of discharge) shows no dilated loops of gas-filled bowel. 
of admission, the nasogastric tube was removed, and he was started on a soft diet. He was discharged on the 11th day and continued PD without any problem.

\section{DISCUSSION}

We report a case of acute intestinal obstruction after a colonoscopy in a patient on PD without previous episodes of peritonitis. This patient was successfully treated with decompression and bowel rest. This report suggests that a careful monitoring for any complications after a colonoscopy in PD patients is necessary.

A recent study showed that only $0.31 \%$ suffered complications in the general population with bleeding $(0.22 \%)$, perforation $(0.03 \%)$, and cardiorespiratory complications $(0.06 \%)$ [5]. Although peritonitis without perforation after a colonoscopy has not been reported in the general population, it infrequently has been seen in patients on PD. A previous retrospective study [6] revealed that the average risk of peritonitis after colonoscopy was $6.3 \%$, from a cohort of 77 PD patients after 97 endoscopic procedures. Less commonly, adhesions may form as a result of an intra-abdominal inflammatory conditions, such as peritonitis, appendicitis, or acute diverticulitis [7], and may cause bowel obstruction. However, to the best of our knowledge, acute intestinal obstruction after a colonoscopy without previous peritonitis in patients on PD has not been reported. Moreover, risk factors for the occurrence of ileus after colonoscopy were not clearly reported in the literature.

In the general population, bowel obstruction as a complication of colonoscopy is associated with a history of abdominal surgery. To date, there have been three reported cases of small bowel obstruction after a colonoscopy and all had a history of abdominal surgery [8-10]. However, this patient had no history of abdominal surgery. Another important pathogenesis of adhesive bowel obstruction is believed to result from an inflammatory response of the peritoneum [7]. Theoretically, patients on PD have predisposing factors - impaired systemic immunity and peritoneal local defense - for inflammatory conditions, which together with repeated exposure to dextrose-containing PD fluid facilitate bacterial growth and peritonitis development [11]. However, in this case, the patient had severe ileus after a colonoscopy without acute peritonitis or any other risk factors for ileus, such as previous intestinal surgery or recurrent peritonitis. To the best of our knowledge, this is the first case report presenting severe ileus after a colonoscopy in a stable patient on PD.

The CT of our patient showing a severely dilated bowel above the distal ileum, and a collapsed bowel below, suggests that there may be an adhesion band in the distal ileum. In contrast to congenital adhesions - usually asymptomatic acquired adhesions occur from previous peritoneal injury or intraperitoneal infection, and the majority of adhesions are postsurgical [7]. Patients on PD have many predisposing factors for chronic intraperitoneal inflammatory conditions; therefore, these patients likely have increased risk for adhesion compared with the general population. Repeated exposures to bio-incompatible PD solutions are related to the development of intraperitoneal inflammation [12], and the PD catheter itself is also independently associated with the disruption of peritoneal membrane integrity, which contributes to intraperitoneal adhesion [13]. However, anatomical adhesion band in this case was not confirmed. Rather, the development of ileus after a colonoscopy in this case might have been associated with a large amount of iatrogenic air during the procedure, impaired bowel motility associated with sedative agents, and/or subclinical peritoneal inflammation.

In conclusion, our case suggests that it is not only necessary to prevent peritonitis, but also important to monitor the development of ileus after a colonoscopy in PD patients. Especially in PD patients, it leads to more serious outcomes, including PD technique failure or worse patient outcomes. If a patient on PD has undergone a colonoscopy and develops abdominal pain, it is important to check for the possibility of ileus, perforation, or peritonitis.

\section{ACKNOWLEDGEMENT}

This work was supported by a grant from the Korea Health Technology R\&D Project (A111345); and by a grant from the Korea Health technology R\&D Project through the Korea Health Industry Development Institute (KHIDI) (HI15C0001).

\section{CONFLICT OF INTEREST}

No potential conflict of interest relevant to this article was reported. 


\section{REFERENCES}

1. Ko HH, Jamieson T, Bressler B. Acute pancreatitis and ileus post colonoscopy. Can J Gastroenterol 2009;23:551-3.

2. Church J. Complication. In: Waye JD, Rex DK, Williams CB, editors. Colonoscopy: principles and practice. Malden, Mass.: Blackwell; 2003. p. 170-80.

3. Wu HH, Li IJ, Weng CH, Lee CC, Chen YC, Chang MY, et al. Prophylactic antibiotics for endoscopy-associated peritonitis in peritoneal dialysis patients. PLoS One 2013;8: e71532.

4. Poortvliet W, Selten HP, Raasveld MH, Klemt-Kropp M. CAPD peritonitis after colonoscopy: follow the guidelines. Neth J Med 2010;68:377-8.

5. Crispin A, Birkner B, Munte A, Nusko G, Mansmann U. Process quality and incidence of acute complications in a series of more than 230,000 outpatient colonoscopies. Endoscopy 2009;41:1018-25.

6. Piraino B, Bailie GR, Bernardini J, Boeschoten E, Gupta A, Holmes C, et al. Peritoneal dialysis-related infections recommendations: 2005 update. Perit Dial Int 2005;25:107-31.

7. Liakakos T, Thomakos N, Fine PM, Dervenis C, Young RL.
Peritoneal adhesions: etiology, pathophysiology, and clinical significance. Recent advances in prevention and management. Dig Surg 2001;18:260-73.

8. Kim HJ, Lee IT, Yok EG, Lee SJ, Lee SH, Kim DS, et al. Intestinal obstruction aggravated by colonoscopy: a case report. J Korean Soc Coloproctol 2009;25:56-8. Korean.

9. Hunter IA, Sarkar R, Smith AM. Small bowel obstruction complicating colonoscopy: a case report. J Med Case Rep 2008;2:179.

10. González Ramírez A, Avila S, López-Rosés L, Lancho A, Santos E, Soto S, et al. Small bowel obstruction and perforation after colonoscopy. Endoscopy 2003;35:192.

11. Yip T, Tse KC, Lam MF, Cheng SW, Lui SL, Tang S, et al. Risks and outcomes of peritonitis after flexible colonoscopy in CAPD patients. Perit Dial Int 2007;27:560-4.

12. Williams JD, Craig KJ, Topley N, Von Ruhland C, Fallon M, Newman GR, et al. Morphologic changes in the peritoneal membrane of patients with renal disease. J Am Soc Nephrol 2002;13:470-9.

13. Flessner MF, Credit K, Henderson K, Vanpelt HM, Potter $\mathrm{R}, \mathrm{He} \mathrm{Z}$, et al. Peritoneal changes after exposure to sterile solutions by catheter. J Am Soc Nephrol 2007;18:2294-302. 